\title{
Time-Varying Radome Slope Estimation for Passive Homing Anti-Ship Missiles
}

\author{
Won-Sang Ra, Sejoon Ahn, Yunha Lee and Ick-Ho Whang
}

\begin{abstract}
This paper addresses a time-varying radome slope (RS) estimation problem for passive homing anti-ship missiles. Apart from conventional approaches, the non-linear characteristic of radome aberration error is taken into account for modeling the RS dynamics. In addition, it is shown that the acceleration dither is necessary for ensuring the observability of the RS estimation with passive seeker measurements. Based on this observation, a linear RS measurement equation is set up by analyzing the seeker response to the high frequency acceleration dither. Thus, the RS estimation problem can be easily resolved by designing a time-varying Kalman filter. Since the proposed approach adopts a simple linear filter structure, it is suitable for in-flight real-time RS estimation. Through the computer simulation for a a typical ASM-target engagement scenario, the usefulness of the suggested scheme is demonstrated.
\end{abstract}

\section{INTRODUCTION}

Most RF homing missiles adopt a tangent-ogive shaped radome in order to protect their seeker antenna from the mechanical damage and to reduce the aerodynamic drag as well [1], [2]. Provided that a radome is non-hemispheric, the radio wave would be refracted by the radome with incidence angle dependent permittivity. That is, the seeker measurements contain unwanted errors which vary with the angular position of a target. In detail, the radome refraction results in line-of-sight (LOS) angle and rate measurement errors which are described by the radome aberration error (RAE) and radome slope (RS), respectively [3].

The proportional navigation $(\mathrm{PN})$ is one of the most widely used guidance laws because of its simple structure. The PN guidance command can be easily calculated using the LOS rate and the closing velocity provided by a RF seeker. Since the homing performance of a PN guided missile mainly relies on the accuracy of the LOS rate information, the slope of RAE, often called the RS, may cause severe degradation of the homing guidance performance [4], [5], [12]. For this reason, it is essential to develop a RS estimation method to enhance the overall guidance performance of a RF homing missile.

The existing RS estimation techniques can be classified into two categories according to its implementation scheme;

This work was supported by Agency for Defense Development (Project No.: 311225-914022301).

W.-S. Ra is with School of Mechanical and Control Engineering, Handong Global University, 558 Handong-ro, Heunghae-eup, Buk-gu, Pohang, Gyeongbuk, 37554 South Korea, wonsang@ handong.edu

S. Ahn and I.-H. Whang are with Agency for Defense Development, P.O. Box 35, Yuseong, Daejeon, 34186 South Korea, \{sejoon86@gmail.com, ickho@add.re.kr\}

Y. Lee is with School of Aerospace, Transport and Manufacturing, Cranfield University, Cranfield, MK43 0AL United Kingdom, yunha.leedcranfield.ac.uk off-line and on-line methodologies. Off-line methods try to model the RS characteristics through theoretical analysis and/or experiment for an arbitrary chosen radome sample [6], [7], [8]. Once the nominal feature of the radome is obtained, it is stored on the on-board computer of a missile and is used for compensating the RS in flight. While this method requires much time and effort for improving the accuracy of the RS model, it might be very restrictive in practice. This is because the RS characteristics modeled by a ground test is different from that observed during flight due to aerodynamic heating.

To make up the flaws of the off-line method, it is desirable to develop an on-line RAE estimation scheme [9]. So far, under the standing assumption that the seeker provides range and LOS information, the interactive multi-model (IMM) method has been studied [10], [11]. This approach designed a set of psuedo-linear Kalman filters for different RS hypotheses. Since each hypothesis probability can be calculated by the corresponding pseudo-linear subfilter, the RS estimate becomes the probabilistically weighted sum of RS estimates from the subfilters. However, its RS estimation performance tends to be severely deteriorated when the range information is not available due to RF jamming [9]. The lack of observability on the RS has been recognized as the key technical issue of a RS estimation for passive homing missiles.

As a practical way to effectively cope with the observability problem, the use of the acceleration dither has been attempted [12]. This methods is based on the fact that the LOS dynamics has a relatively low frequency bandwidth compared to the high frequency acceleration dither. Once the seeker response to the intentional dither is modeled as a transfer function, the RS can be calculated using this model and the filtered outputs of the seeker. For its simple structure, this approach is suitable for real-time implementation. However, it may suffer from difficulties in estimating the time-varying RS as it requires the RS to be a constant.

In order to overcome the limitations of the previous studies, in this paper, a new time-varying RS estimator is proposed for passive homing ASM (anti-ship missile)s. The RS dynamics model is derived by taking the non-linear RAE characteristics expressed as a composite sinusoid of the look angle. It is explained why the acceleration dither is effective to ensure the observability on the RS. Assuming that the high frequency acceleration dither is issued, the seeker response is analyzed to derive a linear RS measurement model. Based on this analysis result, it is shown that the band-pass filtered look angle of a seeker is proportional to the RS. 


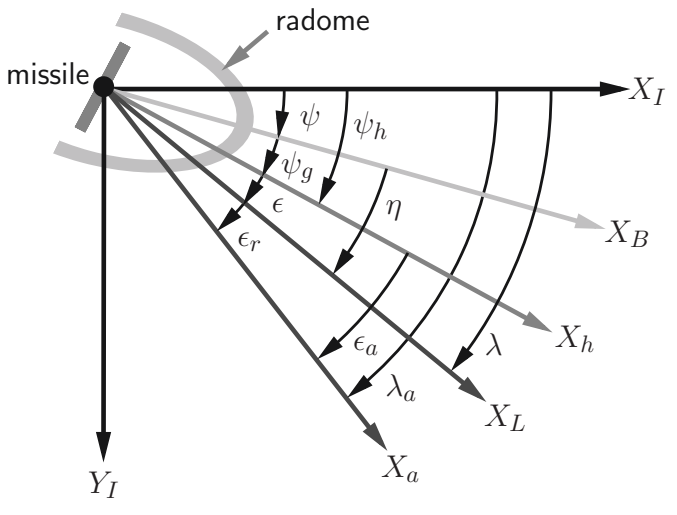

Fig. 1. Missile-target relative geometry

Therefore, the RS estimation problem can be easily resolved by designing a time-varying Kalman filter for these models. In contrast with the existing method, the proposed method is applicable even when the RS is inherently time-varying and nonlinear. Furthermore, the proposed RS estimator is very practical because it has a second order recursive linear filter structure and does not require the a prior knowledge on the seeker transfer function. Through computer simulations, it is shown that the satisfactory homing guidance performance can be achieved with the help of the proposed filter even when the RAE exists.

\section{Time-VARYing RAE Estimation Problem}

\section{A. RAE Properties}

Before designing a RS estimator, let us first review the typical RAE characteristics of a RF seeker. To do this, consider the missile-target relative geometry in horizontal plane depicted in Fig. 1. The variables used in the figure are listed below.

$\begin{array}{cl}\left(X_{I}, Y_{I}\right) & \text { inertial frame } \\ X_{B} & \text { missile nose direction } \\ X_{h} & \text { antenna heading } \\ X_{L} & \text { true target direction } \\ X_{a} & \text { apparent target direction } \\ \psi & \text { missile body angle } \\ \psi_{g} & \text { gimbal angle } \\ \psi_{h} & \text { antenna heading angle } \\ \lambda & \text { LOS angle } \\ \lambda_{a} & \text { apparent LOS angle } \\ \eta & \text { look angle } \eta \triangleq \lambda-\psi \\ \epsilon & \text { boresight error angle } \\ \epsilon_{r} & \text { RAE } \\ \epsilon_{a} & \text { apparent boresight error angle }\end{array}$

From Fig. 1, it is obvious that the following equation holds.

$$
\lambda=\lambda_{a}-\epsilon_{r}=\psi+\psi_{g}+\epsilon
$$

Figure 2 shows the RAE $\epsilon_{r}$ of a non-hemispheric radome. In the figure, a unknown constant $\alpha$ is introduced to compare the magnitudes of different RAE models. It is reasonable to

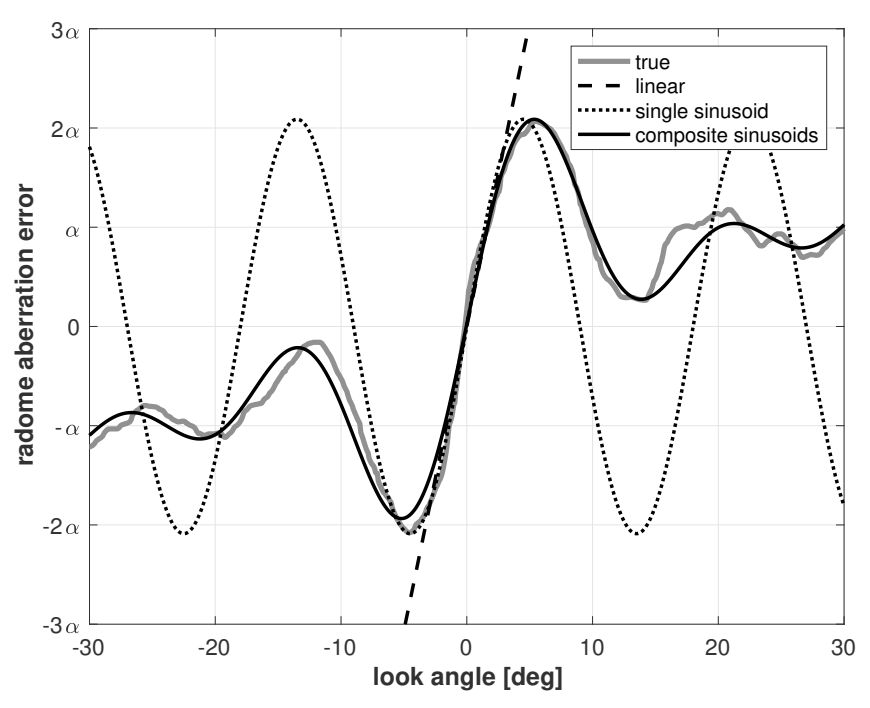

Fig. 2. Time-varying RAE model

model the RAE as a composite sinusoid of the look angle $\eta$.

$$
\epsilon_{r}=\sum_{n}\left[a_{n} \cos \left(n \omega_{o} \eta\right)+b_{n} \sin \left(n \omega_{o} \eta\right)\right],
$$

where $a_{n}$ and $b_{n}$ are arbitrary constants.

Without loss of generality, (2) can be approximated as a sinusoidal function within the $\eta$-range of interest.

$$
\epsilon_{r} \approx \epsilon_{r}^{o} \sin \left(\omega_{o} \eta+\phi^{o}\right), \quad \omega_{o}=\frac{2 \pi}{P^{o}}
$$

In the above, $\epsilon_{r}^{o}$ is the magnitude of RAE angle, $P^{o}$ is the RAE period with respect to the look angle $\eta$ and $\phi^{\circ}$ is the phase shift. Since $P^{o}$ mainly depends on the radome shape, it can be predetermined by a theoretical analysis or a series of experiments. On the other hand, $\epsilon_{r}^{o}$ and $\phi^{o}$ are unknown in general because they are affected by the flight condition. Moreover, the RS is defined as the derivative of the RAE $\epsilon_{r}$ with respect to the look angle $\eta$.

$$
K_{s} \triangleq \frac{d \epsilon_{r}}{d \eta}=K_{s}^{o} \cos \left(\omega_{o} \eta+\phi^{o}\right), K_{s}^{o} \triangleq \omega_{o} \epsilon_{r}^{o} .
$$

As a special case, if $\eta \ll 1$ is satisfied, (3) and (4) reduce to the conventional linear RS estimation.

$$
\epsilon_{r} \approx K_{s} \eta, \quad K_{s} \approx \omega_{o} \epsilon_{r}^{o}=K_{s}^{o}
$$

\section{B. Effectiveness of Dither for RS Estimation}

Note that, while the passive measurements (apparent LOS angle and rate) of a seeker are directly affected by the RAE, its active measurements (range or closing velocity) are insensitive to the RAE. This implies that the target state estimates calculated using the passive measurements differs from those using active measurements. Ironically, this discrepancy plays an important role to enhance the observability on the RS in active homing mode. The RS estimate can be determined as a minimizing solution of this discrepancy. This is the reason why the existing IMM filter based RS estimator works well when the active measurements are provided by a seeker. Unfortunately, this concept cannot be applied for designing a 
RS estimator in passive homing mode, hence an another way to ensure the observability should be considered. Without it, it is clear that the RS estimation cannot be solved by means of a state estimation theory itself.

Underlying idea to tackle the observability issue in passive homing mode is to utilize the fact that the RAE $\epsilon_{r}$ is described as a function of the look angle $\eta=\lambda-\psi$. As shown in Fig. 1, the look angle variation causes the apparent LOS angle variation, hence the RAE $\epsilon_{r}$ can be calculated by analyzing these information. However, this idea is difficult to realize because the look angle $\eta$ is not measurable from a seeker. At this point, the usefulness of the dithering technique can be found.

In order to outline the proposed approach, let us recall that the translation motion of a missile is much slower than the its angular motion in general. Moreover, it is also assumed that the motion of a target is ignorable compared to ASMs. Under these conditions, it is obvious that the LOS angle is slightly changed even when the missile moves very long distance. Thus, the LOS angle is regarded as a slowly varying parameter. Since the translation motion is negligible in short time period, the following approximation makes sense for the increment of the LOS angle.

$$
\Delta \lambda=\lambda^{2}-\lambda^{1} \approx 0
$$

where $\xi^{i}$ denotes the quantity $\xi\left(t_{i}\right)$ defined at the time $t_{i}$. On the other hand, the missile yaw attitude $\psi$ changes quickly when the angular motion of a missile exists. Therefore, the the incremental look angle can be successfully approximated using the missile attitude available from inertial navigation system (INS) unit.

$$
\Delta \eta=\eta^{2}-\eta^{1} \approx-\left(\psi^{2}-\psi^{1}\right)=-\Delta \psi .
$$

As the similar way, we have

$$
\begin{aligned}
\Delta \lambda_{a} & =\lambda_{a}^{2}-\lambda_{a}^{1}=\left(\lambda^{2}-\lambda^{1}\right)+\left(\epsilon_{r}^{2}-\epsilon_{r}^{1}\right) \\
& \approx \epsilon_{r}^{2}-\epsilon_{r}^{1}=\Delta \epsilon_{r} .
\end{aligned}
$$

Substituting (7) and (8) for (4) yields the following equation involving the RS.

$$
K_{s}=\frac{\partial \epsilon_{r}}{\partial \eta} \approx-\frac{\Delta \lambda_{a}}{\Delta \psi}
$$

From (9), the RS can be obtained by observing the increment of the apparent LOS angle to the missile attitude variation. That is, in the case of the passive homing, the intentional angular motion of a missile is necessary to ensure the observability on the RS.

\section{RS Measurement Model}

At this point, it is noteworthy that the change of the look angle $\eta$ induced by the missile acceleration. This implies that the intentional acceleration dither might be very helpful for ensuring the observability on RS in passive homing mode [12]. Since the acceleration dither has a known frequency higher than the bandwidth of the missile dynamics, it has little effect on the homing trajectory. For this reason, in our problem setting, it is assumed that the acceleration dither $d(t)$ is intentionally added to the nominal guidance command.

$$
d(t)=A_{d} \sin \left(\omega_{d} t\right)
$$

In (10), $A_{d}$ and $\omega_{d}$ are the prescribed dither amplitude and frequency, respectively.

Now, the measurement equation of RS can be easily derived by analyzing the seeker response to the acceleration dither $d(t)$ in (10). Fig. 3 shows the seeker control loop where the dotted lines indicate the additional parts necessary for implementing our RS estimator. The seeker output $x$ is perturbed around its nominal value $x^{n}$ by the amount of $x^{d}$ due to the acceleration dither. That is, under the assumption that the seeker is a linear system, the internal signal $x$ of the seeker is decomposed as follows:

$$
x=x^{n}+x^{d}
$$

By the definition of (11), the LOS angle $\lambda$ in (1) can be rewritten as

$$
\begin{aligned}
\lambda^{n}+\lambda^{d}= & \psi^{n}+\psi^{d}+\psi_{g}^{n}+\psi_{g}^{d}+\epsilon_{a}^{n}+\epsilon_{a}^{d} \\
& -\epsilon_{r}^{o} \sin \left(\omega_{o} \eta^{n}+\omega_{o} \eta^{d}+\phi^{o}\right) .
\end{aligned}
$$

If the magnitude of the acceleration dither $d(t)$ is small, the changes of the LOS and the yaw attitude angle due to $d(t)$ are negligible, $\left|\psi^{d}\right| \ll 1$ and $\left|\lambda^{d}\right| \ll 1$. Therefore, by the small angle approximation, eq. (12) can be approximated as

$$
\begin{aligned}
& \lambda \approx \psi^{n}+\psi^{d}+\psi_{g}^{n}+\psi_{g}^{d}+\epsilon_{a}^{n}+\epsilon_{a}^{d} \\
&-\epsilon_{r}^{o} \sin \left(\omega_{o} \eta^{n}\right)+\underbrace{K_{s}^{o} \cos \left(\omega_{o} \eta^{n}+\phi^{o}\right)}_{K_{s}^{n}} \psi^{d} .
\end{aligned}
$$

In general, the dither frequency is already known and higher than the bandwidth of the angular measurements. Hence, one can readily extract the response to the acceleration dither, $x^{d}$, from the seeker output, $x$, by using the band-pass filter $B_{f}(s)$ whose center frequency is equal to the dither frequency, $\omega_{d}$. By applying band-pass filter $B_{f}(s)$ to (13), a linear relation between the RS and the seeker outputs can be obtained.

$$
\psi^{d}+\psi_{g}^{d}+\epsilon_{a}^{d}=-K_{s}^{n} \cdot \psi^{d}
$$

where $\psi, \psi_{g}$ and $\epsilon_{a}$ are measured by the inertial navigation system, the gimbal potentiometer and the seeker discriminator, respectively. It should be noted that measurement noise is added to each measured signals. Hence,

$$
\tilde{\psi}=\psi+\delta \psi, \quad \tilde{\psi}_{g}=\psi_{g}+\delta \psi_{g}, \quad \tilde{\epsilon}_{a}=\epsilon_{a}+\delta \epsilon_{a}
$$

By taking into account the fact that the missile body angle measurement error $\delta \psi$ is negligible and is substituting (15) into (14), the meausrement equation for the RS estimator can be derived as

$$
\psi^{d}+\tilde{\psi}_{g}^{d}+\tilde{\epsilon}_{a}^{d}=-\hat{K}_{s}^{n} \cdot \psi^{d} .
$$




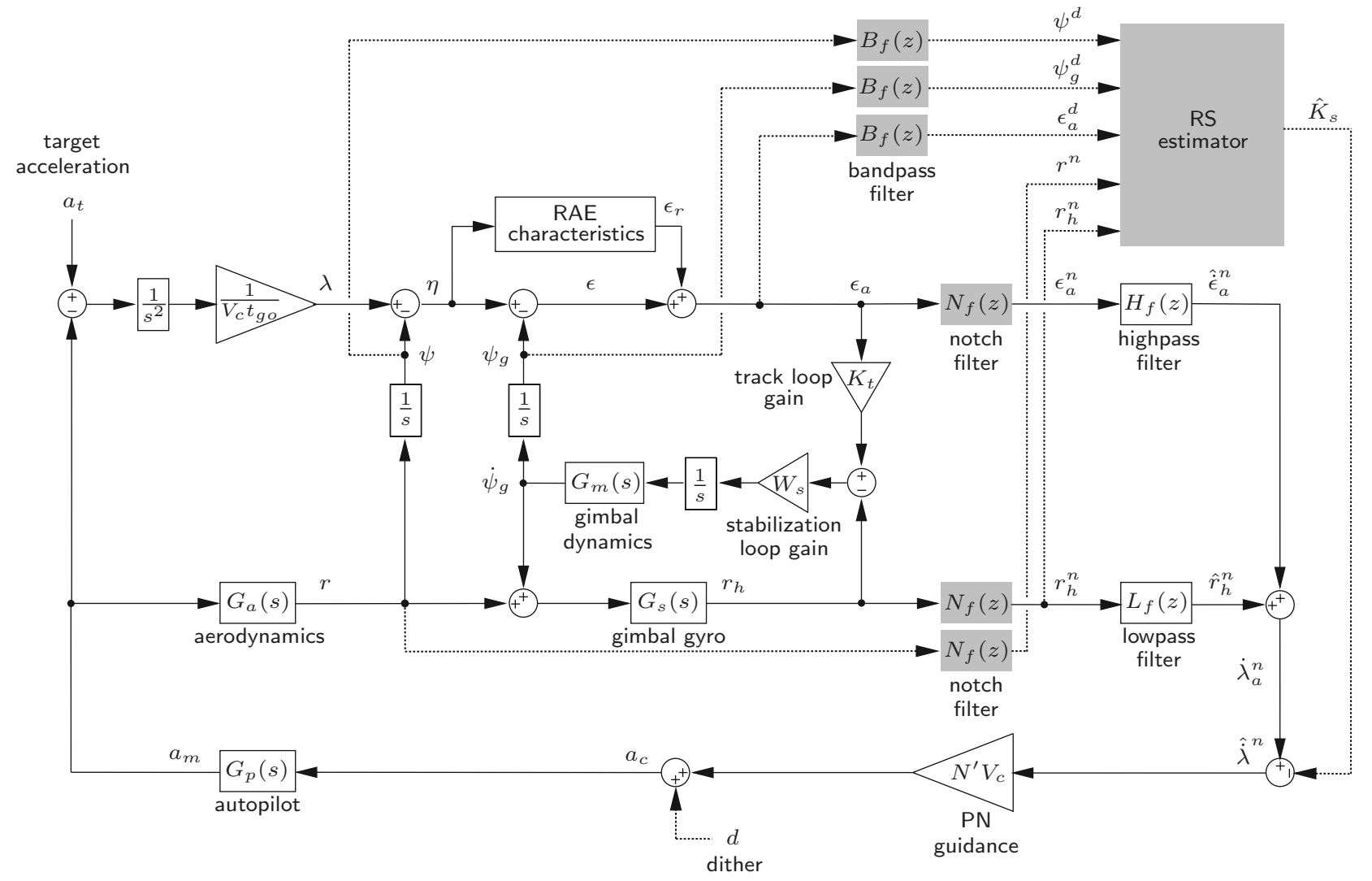

Fig. 3. Homing guidance loop

\section{RS Dynamics Model}

Taking the time varying nature of the RAE into account, let us derive the dynamics of the nominal RS $K_{s}^{n}$. Using eq. (4) and the definition of (11), one gets

$$
\begin{aligned}
& \dot{K}_{s}^{n}=-\epsilon_{r}^{o} \sin \left(\omega_{o} \eta^{n}+\phi^{o}\right) \omega_{o} \dot{\eta}^{n}, \\
& \ddot{K}_{s}^{n}=-\epsilon_{r}^{o} \cos \left(\omega_{o} \eta^{n}+\phi^{o}\right)\left(\omega_{o} \dot{\eta}^{n}\right)^{2} \\
& \quad-\epsilon_{r}^{o} \sin \left(\omega_{o} \eta^{n}+\phi^{o}\right) \omega_{o} \ddot{\eta}^{n} .
\end{aligned}
$$

If the seeker's tracking loop is properly working in a homing phase, the boresight error angle $\epsilon$ is maintained as a small value. Therefore, without loss of generality, one can assume that $\eta=\lambda-\psi \approx \psi_{g}$. In most ASM homing scenarios, it is reasonable to assume that the rate of look angle can be approximated as a ramp input in short period and the missile's lateral acceleration is slowly varying. For such case, since the angle tracking loop of the seeker is the type 1 system, the approximation $\ddot{\psi}_{g}=\dot{r}_{h}-\dot{r} \approx 0$ makes sense. Based on these results, (17) can be rewritten as

$$
\ddot{K}_{s}^{n} \approx-K_{s}^{n}\left(\omega_{o}\left(r_{h}-r\right)\right)^{2}-\omega_{o} \epsilon_{r}^{n}{\left.\underline{\dot{r}_{h}}-\vec{r}\right) .^{0}}^{0}
$$

\section{RS Estimator DESIGN}

Incorporating the measurement equation (14), with the dynamics equation (18), the continuous time system model can be obtained. After discretization of this model with a sampling period $T_{s}$, one gets the following discrete time system model required for the time-varying RS estimator design.

$$
\left\{\begin{array}{c}
\boldsymbol{x}_{k+1}=F_{k} \boldsymbol{x}_{k}+G_{k} u_{k} \\
y_{k}=H_{k} \boldsymbol{x}_{k}+v_{k}
\end{array}\right.
$$

where the state vector $\boldsymbol{x}$, the measurement $y$ and the relevant matrices are defined as

$$
\begin{gathered}
\boldsymbol{x}=\left[\begin{array}{ll}
K_{s}^{n} & \dot{K}_{s}^{n}
\end{array}\right]^{T}, y=-\left(\psi^{d}+\tilde{\psi}_{g}^{d}+\tilde{\epsilon}_{a}^{d}\right), \\
F=\left[\begin{array}{cc}
1 & T_{s} \\
-\left(\omega_{o}\left(\tilde{r}_{h}^{n}-\tilde{r}^{n}\right)\right)^{2} T_{s} & 1
\end{array}\right], \\
G=\left[\begin{array}{ll}
0 & 1
\end{array}\right]^{T}, \quad H=\left[\begin{array}{ll}
\psi^{d} & 0
\end{array}\right] .
\end{gathered}
$$

In equation (19), the process noise $u_{k}$, reflecting the modeling uncertainty contained in the RS dynamics, and the measurement noise $v_{k}$ are assumed to be zero-mean, white noise sequences with variances $Q_{k}$ and $R_{k}$ respectively. $\tilde{r}_{h}^{n}$ and $\tilde{r}^{n}$ in the system matrix $F$ are the notch filtered result of the gimbal gyro measurement and the angular rate measurement of the ASM respectively. $\tilde{\psi}^{d}, \tilde{\psi}_{g}^{d}$ and $\tilde{\psi}_{a}^{d}$ in the measurement matrix $H$ is also the attitude of the ASM, the gimbal angle of the seeker and BSE measurement, respectively, through the band-pass filter.

By applying the Kalman filter for the discrete-time system model (19), the filter recursion for estimating time-varying $\mathrm{RS}$ is obtained. 
(measurement update)

$$
\begin{aligned}
\hat{\boldsymbol{x}}_{k \mid k} & =\hat{\boldsymbol{x}}_{k \mid k-1}+K_{f, k}\left(y_{k}-H_{k} \hat{\boldsymbol{x}}_{k \mid k-1}\right) \\
K_{f, k} & =P_{k \mid k-1} H_{k}^{T} R_{k}^{-1} \\
P_{k \mid k}^{-1} & =P_{k \mid k-1}^{-1}+H_{k}^{T} R_{k}^{-1} H_{k}
\end{aligned}
$$

(time update)

$$
\begin{aligned}
& \hat{\boldsymbol{x}}_{k+1 \mid k}=F_{k} \hat{\boldsymbol{x}}_{k \mid k} \\
& P_{k+1 \mid k}=F_{k} P_{k \mid k-1} F_{k}^{T}+G_{k} Q_{k} G_{k}^{T} \\
& \text { IV. PERFORMANCE EVALUATION }
\end{aligned}
$$

\section{PERformance EVAluation}

To evaluate the usefulness of the proposed time-varying RS estimator, simulations for typical ASM engagement scenarios are carried out. The RS estimation performance is analyzed by running 100 Monte Carlo trials.

For the purpose of performance comparison, our method is tested together with the previous method in [12] which can be expressed as follows:

$$
\begin{aligned}
& \tilde{K}_{s}^{n}=L_{f}\left\{-\frac{\psi^{d}+\tilde{\psi}_{g}^{d}+\tilde{\epsilon}_{a}^{d}}{\psi^{d}}\right\}, \\
& \left.\hat{K}_{s}^{n}=\operatorname{sat}\left(\tilde{K}_{s}^{n}, K_{s}^{m}\right)\right)
\end{aligned}
$$

In the above, $L_{f}(\bullet)$ and $\operatorname{sat}(\bullet)$ denote a low-pass filter and a saturation function, respectively. These are required for suppressing RS estimation values to become unrealistically large. $K_{s}^{m}$ is the allowable maximum value of the estimated RS. The transfer function of the low-pass filter is defined by

$$
L_{f}(s)=\frac{1}{T_{L P F} s+1}, T_{L P F}=0.5[\mathrm{sec}], K_{s}^{m}=0.1
$$

TABLE I

SIMULATION CONDITION

\begin{tabular}{c|l}
\hline \hline item & \multicolumn{1}{c}{ parameter } \\
\hline \multirow{3}{*}{ target } & $\vec{R}_{t}^{I}(0)=(0.0)[\mathrm{km}]$, \\
& $\vec{V}_{t}^{I}(0)=V_{t}\left(\cos \left(\psi_{t}(0)\right), \sin \left(\psi_{t}(0)\right)\right)$, \\
& $V_{t}=20[\mathrm{~m} / \mathrm{s}], \psi_{t}(0)=135^{\circ}$ \\
& $\vec{R}_{m}^{I}(0)=(-20,0)[\mathrm{km}]$, \\
& $\vec{V}_{m}^{I}(0)=V_{m}(\cos \psi(0), \sin \psi(0))$, \\
& $V_{m}(0)=700[\mathrm{~m} / \mathrm{s}], \psi(0)=30^{\circ}$, \\
& $G_{a}(s)=\frac{T_{\alpha} s+1}{V_{m}}, T_{\alpha}=2.0[\mathrm{sec}]$ \\
missile & $G_{p}(s)=\left(\frac{1}{1+s T / 5}\right), T=0.5[\mathrm{sec}]$ \\
\hline autopilot & $N=3, A_{d}=1[\mathrm{~g}], \omega_{d}=2.5[\mathrm{~Hz}]$ \\
\hline guidance & $K_{t}=8[\mathrm{rad} / \mathrm{s}]:$ track loop gain \\
& $W_{s}=60[\mathrm{rad} / \mathrm{s}]:$ stab. loop gain \\
& $K_{k}^{o}=0.08, P^{o}=40^{\circ}$ \\
\hline seeker & $\sigma_{r_{h}}=0.3^{\circ}, \sigma_{r}=0.3^{\circ}, \sigma_{\psi_{g}}=0.01^{\circ}$ \\
& $\sigma_{\psi}=0.01^{\circ}, \sigma_{\epsilon}=0.02^{\circ}$ \\
\hline noise & $\hat{\boldsymbol{x}}_{0 \mid-1}=0^{2 \times 1}, P_{0 \mid-1}=$ diag $\left(0.1^{2}, 0.5^{2}\right)$, \\
statistics & $Q=0.1^{2}, R=\left(0.05^{\circ}\right)^{2}, T_{s}=2.5[\mathrm{~ms}]$ \\
\hline \multirow{2}{*}{ RS estimator } &
\end{tabular}

The RS estimators based on the dither technique inevitably require the band-pass filtered signals, $\psi^{d}$ and $\tilde{\psi}_{g}^{d}$. To do this, a second order Butterworth band-pass filter $B_{f}(s)$ is used in the simulation. Note that, to secure the smooth transition from midcourse guidance to homing guidance, it is desirable to execute our RS estimator after the band-pass filter converges. In our case, the convergence time of the band-pass filter is set as $5[\mathrm{sec}]$. Fig. 3 shows the overall block diagram of the homing guidance loop including an RS estimator.

To investigate the effect of the RS's sign, the case when $K_{s}^{\circ}=0.08$ is considered in the simulation and the result is depicted in Fig. 4. From the figure, it is clear that the proposed estimator provides better RS estimation performance than the previous method. Our method maintains satisfactory RS estimation performance; its estimation error mean is less than $2 \%$ and its standard deviation is about $1 \%$ after convergence. On the contrary, the previous scheme provides unreliable RS estimation performance. In transient period, its estimation error is severely fluctuating. Furthermore, its estimation error standard deviation is larger than $4 \sim 5 \%$ during homing guidance. This means that the previous method cannot be successful in estimating the time-varying RS. As shown in Fig. 4, while the proposed filter successfully copes with the RS varies with the look angle, the previous approach does not. This is because the proposed RS estimator is designed using the RS dynamics derived in Section III.D.

Now let us use the estimated RS $\hat{K}_{s}^{n}$ to compensate the RAE-contaminated LOS rate, use the compensated LOS rate in the homing loop, analyze the homing performance on the basis of miss distance and evaluate the usefulness of the proposed RS estimator. From the angular relation given in section 2.1, the following equation holds.

$$
\dot{\lambda}_{a}^{n}=\dot{\lambda}^{n}+\dot{\epsilon}_{r}^{n}=\dot{\lambda}^{n}+K_{s}^{n}\left(\dot{\lambda}^{n}-\dot{\psi}^{n}\right)
$$

Therefore, the compensated LOS rate can be expressed as

$$
\hat{\dot{\lambda}}^{n}=\frac{\tilde{\dot{\lambda}}_{a}^{n}+\hat{K}_{s}^{n} \tilde{r}^{n}}{1+\hat{K}_{s}^{n}} \text {. }
$$

The miss distance is also analyzed for case that the RS has positive sign $\left(K_{s}^{n}=0.08\right)$. The simulation results are summarized in Fig. 5. When the LOS rate without RS compensation is used for homing guidance, the miss-distance lies between $6 \sim 7[\mathrm{~m}]$, which is unacceptable to most precision guided ASMs. On the other hand, if the LOS rate measurement of the seeker is compensated by using the RS estimate obtained from our filter, the guidance performance is dramatically improved even when the radome aberration error exists. Provided that the initial range between the ASM and the target is larger than $13[\mathrm{~km}]$, the miss-distance is under $1[\mathrm{~m}]$ which is comparable to the ideal homing guidance performance when there is no radome aberration error. In spite of using our RS estimator, the guidance performance could be degraded when the enough flight time is not given for homing guidance. Nevertheless, the homing guidance loop with our RS estimator provides better performance than that using radome error contaminated LOS rate. 


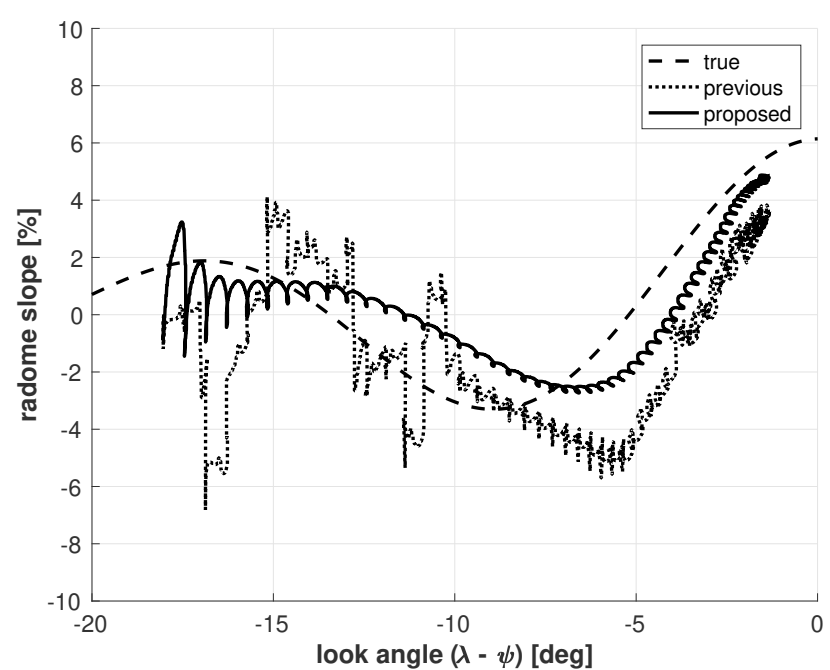

(a)

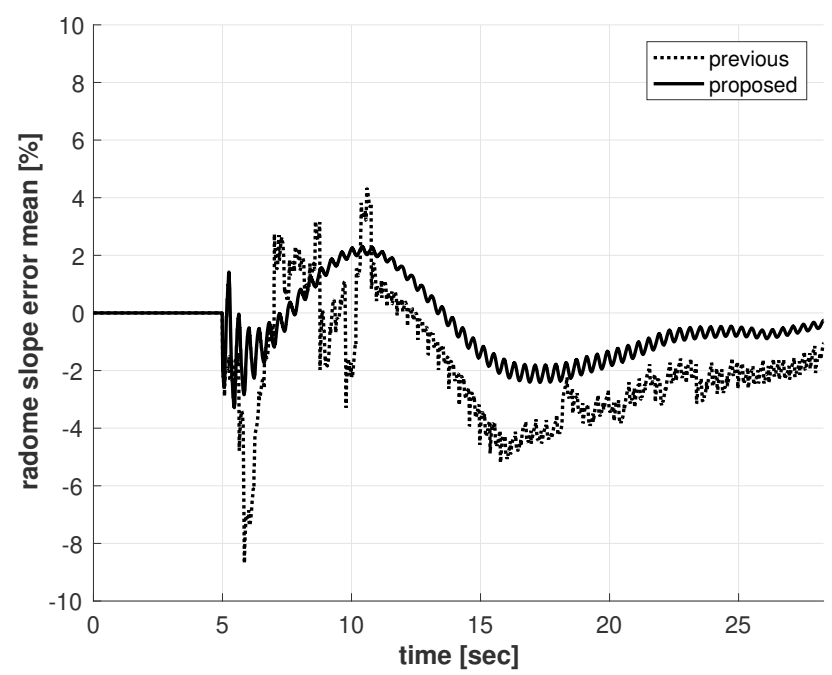

(b)



(c)

Fig. 4. RS estimation performance $\left(K_{s}=0.08\right)$

\section{CONCLUSION}

A new RS estimation filter for passive homing ASMs has been proposed. In order to accommodate the time-varying

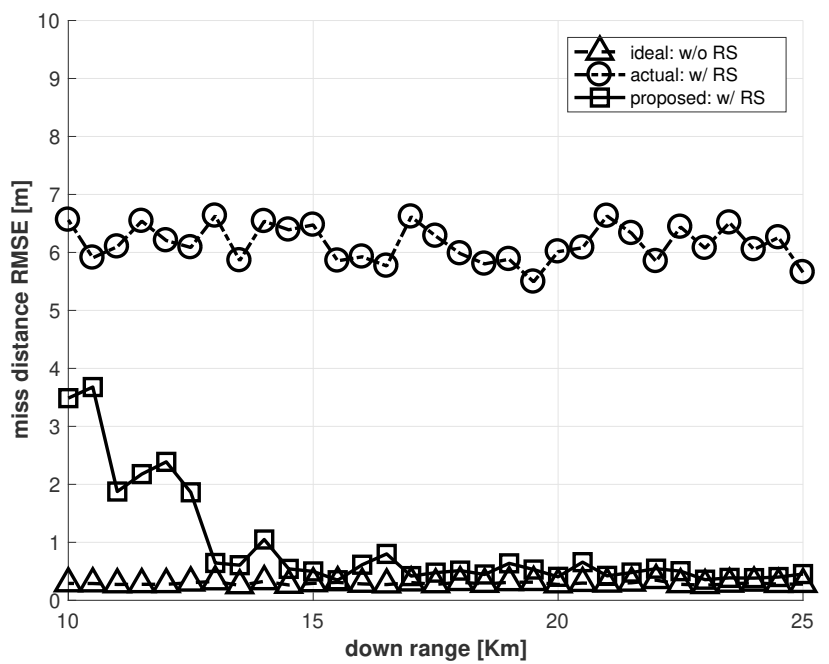

Fig. 5. Guidance performance comparison

characteristics of the RS, the RAE angle has been approximated as a sinusoidal function of the look angle. Using this approximation, the RS dynamics was derived. Under the assumption that the acceleration dither is applied for ensuring the observability on RS estimation, a measurement equation is obtained using the band-pass filtered seeker outputs. From the derived radome dynamics and measurement equation, a recursive RS estimator was designed. From simulations, it has been confirmed that the RMSE of the RS estimate provided by the proposed method is less than $3 \%$. It is shown that the proposed RS estimator is very useful to improve the homing guidance performance of PN guided ASMs.

\section{REFERENCES}

[1] P. Garnell, and D.J. East, Guided Weapon Control Systems, Pergamon Press, New York, 1980.

[2] N.F. Palumbo, R.A. Blauwkamp, and J.M. Lloyd, and D.J. East, "Basic Principles of Homing Guidance", Johns Hopkins APL Technical Digest, 29(1), 2010, pp.25-41

[3] T. Murray, "Correlation of Linear and Nonlinear Radome Error Induced Miss Distance Predictions", American Control Conf., 1984, pp.743-750.

[4] F.W. Nesline, and P. Zarchan, "Radome Induced Miss Distance in Aerodynamically Controlled Homing Missiles", AIAA Guidance and Control Conference, 1984, pp.84-1845.

[5] W.R. Yueh, and C.F. Lin, "Guidance Performance Analysis with Inflight Radome Error Calibration", J. Guidance, Control, and Dynamics, 8(5), 1985, pp.666-669.

[6] D. Paris, "Computer-Aided Radome Analysis", IEEE Trans. Antennas and Propagation, 18(1), 1970, pp.7-15.

[7] J. Chang, and K. Chan, "Analysis of a Two-Dimensional Radome of Arbitrarily Curved Surface", IEEE Trans. Antennas and Propagation, 38(10), 1990, pp.1565-1568.

[8] H. Lee, and H. Park, "Prediction of Radome Bore-Sight Errors Using a Projected Image of Source Distributions", Progress In Electromagnetics Research, 92, 2009, pp.181-194.

[9] R. Kumar, Defense of Surface Ships Against Anti Ship Missiles, Ph.D. Dissertation, Monterey, California: Naval Postgraduate School, 1990.

[10] J.M. Lin, and Y.F. Chau, "Radome Slope Compensation Using Multiple-Model Kalman Filters", J. Guidance, Control, and Dynamics, 18(3), 1995, pp.637-640.

[11] T.L. Song, D.G. Lee, and S.J. Shin, "Active Homing Performance Enhancement with Multiple Model Radome Slope Estimation", $J$ Aerospace Engineering, 219(3), 2005, pp.217-224.

[12] P. Zarchan, and H. Gratt, "Adaptive Radome Compensation Using Dither", J. Guidance, Control, and Dynamics, 22(1), 1999, pp.51-57. 


\title{
Time-varying radome slope estimation for passive homing anti-ship missiles
}

\author{
Ra, Won-Sang
}

IEEE

Ra W-S, Ahn S, Lee Y \& Whang I-H (2020) Time-varying radome slope estimation for passive homing anti-ship missiles. In: 2019 IEEE 58th Conference on Decision and Control (CDC), 11-13 December 2019, Nice, France https://doi.org/10.1109/CDC40024.2019.9029281

Downloaded from Cranfield Library Services E-Repository 